Results A total of 12983 colonoscopies were performed during this period. 547 (age range 30-98 years) cases of CRC were diagnosed, $54 \%$ of them were males and 48 (8.7\%) were less than 50 years of age. Clinical presentation and other characteristics were compared between young and old illustrated in table 1.

Most common clinical presentations in young were; Anaemia (40\%) Rectal bleeding(40\%), change in bowel habits$\mathrm{CIBH}(12 \%)$ while older patients were presented with anaemia(22\%), CIBH (19\%), rectal bleeding (21\%) and abnormal imaging(19\%). Significant proportion of young patients showed thrombocytosis when compared to older individuals (23\% vs $14 \%, \mathrm{p}=0.03)$.

Most common sites were rectum and SC in both cohorts. In older population $43 \%$ of CRC were in proximal colon while only $14 \%$ in young had a proximal CRC $(p=0.01)$

Majority of the CRC were adenocarcinoma (90\%), and the remainder were anal squamous, neuro endocrine. $4.7 \%$ of cancers were not confirmed by histology.

Significant proportion of patients were diagnosed at an advanced stage (3 and above) $31 \%$ and $32 \%$ in older and younger age group respectively. 59\% of our cohort had elective or emergency surgery while the rest were treated with a palliative intent. One year mortality was $12.5 \%$ and $19 \%$ for young and older cohort respectively.

5 year survival rate was higher in young patients than older $(77.8 \%$ vs $69.2 \%)$.

Conclusions In our cohort younger CRCs presented with anaemia and rectal bleeding while older cohort with $\mathrm{CIBH}$ and anaemia/rectal bleeding. Thrombocytosis was a distinct feature in young CRCs. Even through significant proportion of younger CRCs presented with advanced disease, 5 year survival rate was higher in young.

\section{P319 FAECAL IMMUNOCHEMICAL TESTING: COST EFFECTIVE WAY STRATIFYING SYMPTOMATIC PATIENTS FOR URGENT STRAIGHT TO TEST INVESTIGATION}

Baljit Singh, Jaspreet Seehra*, James Stephenson, Daniel Barnes, Paul Danaher, James Jones, Timothy Osborne, Farah Khasawneh, Elena Pérez Fernández, Ellie WightmanBragg. University Hospitals of Leicester, Leicester, UK

\subsection{6/gutjnl-2020-bsgcampus.393}

Background Quantitative faecal immunochemical test (FIT) offers the opportunity to stratify symptomatic 'high risk' colorectal patients for further investigation.

Method FIT was introduced in primary care to stratify 'high risk' symptomatic patients aged 60 years and above with a change in bowel habit to determine whether an urgent straight to test (STT) CT colonography (CTC) was indicated. All FIT tests were analysed in a national bowel screening hub using the OC-Sensor platform. A result of $\geq 4 \mu \mathrm{gHb} / \mathrm{gFaeces}$, was used as the cut-off. All FIT results were cross referenced with a prospectively maintained colorectal cancer registry to determine the colorectal cancer detection rate (CRC). Data was analysed from February 2018-December 2019.

Results The mean number of total CTC performed per month pre-FIT was 240 (range 185-278) and reduced to 217 (range 183-264) post-implementation $(\mathrm{p}<0.05)$. The number referred under the STT pathway was 167 (range 119-209) reducing to 131 (range 91-153) ( $\mathrm{p}<0.05$ ), however there was a corresponding rise in the number of non-STT referrals

\begin{tabular}{|c|c|c|c|}
\hline $\begin{array}{l}\text { FIT Result } \mu \mathrm{gHb} / \\
\text { gFaeces }\end{array}$ & $\begin{array}{l}\text { Number of Colorectal } \\
\text { Cancers }\end{array}$ & $\begin{array}{l}\text { All } \\
\text { Tests }\end{array}$ & $\begin{array}{l}\text { CRC Detection } \\
\text { Rate }\end{array}$ \\
\hline$<4$ & 10 & 5337 & $0.2 \%$ \\
\hline $4-9.9$ & 5 & 976 & $0.5 \%$ \\
\hline $10-149.9$ & 34 & 1337 & $2.5 \%$ \\
\hline$>150$ & 55 & 313 & $17.6 \%$ \\
\hline Grand Total & 104 & 7963 & $1.3 \%$ \\
\hline
\end{tabular}

from outpatients 73 (range 44-105) to 85 (range 60-111) (p $<0.05)$.

Conclusion FIT has the potential to reduce the burden on secondary care investigations to exclude bowel cancer. Our experience has shown that a conservative FIT level of $<4 \mathrm{ug} / \mathrm{ml}$ has reduced numbers of STT referrals by $22 \%$.

\section{P320 LESSONS FROM PRIMARY CARE: IMPLEMENTING FIT FOR PATIENTS WITH LOWER RISK SYMPTOMS IN NORTH LONDON}

Clare Stephens*, Edward Seward. The Speedwell Practice And NCL-STP, London, UK

\subsection{6/gutjnl-2020-bsgcampus.394}

Introduction GP's need direct access reliable tests to help them hone diagnostic decisions and integrate with secondary care to make best use of limited resources, maximise impact and contribute to resilience in the system. Correct use of Faecal Immunochemical Test (FIT) has the capacity to revolutionise the patient pathway experience and management of colorectal symptoms within primary care. FIT has been proposed by NICE to be the investigation of choice in patients with a 'low risk, but not a no risk' of colorectal cancer (quantified as $0.1-3 \%$ cancer risk). FIT positive patients are advised to be referred up on a 2 week wait (2ww) urgent cancer pathway.

Methods FIT testing in primary care was introduced in North Central London in April 2019. The launch was accompanied by a sector wide education program of talks, information leaflets, and an educational video. Data was collected from a GP practice of 11,440 patients that led the implementation of the test in the local area. Data points used were uptake, use and application of the test, the appropriateness of the pathway followed for that patient and the eventual definitive diagnosis of the patient.

Results Over the 10 month audit time frame, 166 FITs were requested $33(20 \%)$ were positive, i.e. $>10 \mu \mathrm{gHb} / \mathrm{gF}$. FIT tests across the whole STP have climbed from 448/month at the start of the audit period to $1507 /$ month by the end.

Of the 33 FIT positive tests, 2 were diagnosed with colorectal cancer, (6\%) with FIT results of $11 \mathrm{gHb} / \mathrm{gF}$ (rectal cancer) and $20 \mathrm{gHb} / \mathrm{gF}$ (sigmoid cancer).

Of the 33 positive FIT tests; 27 were sent on the $2 \mathrm{ww}$ pathway, 3 further chose a private route for investigation. The remaining 3 were not referred for a range of reasons: 2 patients and their families chose to not to pursue further investigation due to advanced age and frailty. One patient was found to have concurrent Campylobacter, and reassessed by their GP as not requiring onward referral. 
11 patients with a negative FIT tests ranging from 4-8 $\mu \mathrm{gHb} / \mathrm{gF}$ were also sent on into the $2 \mathrm{ww}$ pathway, for a range of clinical reasons, as determined by their GPs as warranting cancer exclusion. Across the audit period none of them were found to have colorectal cancer. A total of 91 patients from the practice were referred on the $2 \mathrm{ww}$ colorectal pathway, FIT referrals were $30 \%$ of this total.

Conclusions The results show that FIT is being utilised increasingly by GPs for the colorectal cancer $2 \mathrm{ww}$ pathway, and that a significant proportion of these are being referred with FIT positivity. Direction of educational resource towards FIT roll out will increase its use in primary care. Detecting cancer through use of FIT in those patients with 'low risk but not no risk' symptoms demonstrates the utility of this pathway.

\section{P321 PRESSURE ON WAITING TIMES OF SURVEILLANCE COLONOSCOPIES REDUCED WITH USE OF A CLINICAL VALIDATION TOOL}

Kelly Stone*, Melanie Shores, Michael Lim, Prashant Kant. York Teaching Hospital Foundation Trust, York, UK

\subsection{6/gutjnl-2020-bsgcampus.395}

Introduction The recent 2019 BSG/ACPGBI evidence based guidelines aim to make surveillance colonoscopy more personalised, ensuring it is recommended for people who need it, and not for those who do not. The updated guidelines have resulted in a change of need and timing for this test.

We chose to study whether the implementation of the new guidelines resulted in a reduction of waiting time breaches and demand for surveillance colonoscopy.

Methods Monthly reports with waiting time breaches were obtained from the information team. All demographic data were then recorded on a separate spreadsheet. A retrospective review of previous colonoscopy report(s), histology and refer$\mathrm{ral} /$ clinic letters was then conducted. The new guidelines were utilised and a local decision tree created. Validated patients fell into 4 outcomes: discharge, continued surveillance, not validated or complex patient requiring consultant input. All discharged patients received a letter and a copy was sent to their GP. A Mann-Whitney U-test was performed to compare groups, a p-value $<0.05$ was deemed significant.

Results Preliminary data was collected for 8 weeks. There were a total of 611 patients. Median age for the study population was 68 (IQR 58-75) years.

After implementation of the decision tree, 123 (20\%) patients were not suitable for validation as they were not polyp surveillance patients. Of the remaining 488 patients, 203 (33.2\%) were suitable for discharged (removed from waiting list or cancelled due to duplicate entries or changed procedures). Only six (3\%) patients from the discharge population made further contact. A further 282 (46.2\%) required continued surveillance (as originally planned or changed to a longer interval. Five patients required input from consultants due to complexity.

Patients that were discharged were older than those that returned for surveillance (73 (IQR 63-79) vs. 68 (IQR 60-74) years, $\mathrm{p}$-value $=0.001$. The overall discharge rate of $33.2 \%$ percent equated to a reduction in demand for 41 colonoscopy lists.
Conclusions The new guidelines have led to a reduction in the number of waiting time breaches. Patient feedback from our process has been positive. Long-term plans are needed to ensure a continued reduction of waiting times going forwards.

\section{P322 IS THERE STILL A PLACE FOR FLEXIBLE SIGMOIDOSCOPY IN THE 'FIT' ERA?}

Mohammed Fakhrul-Aldeen*, Mark Cartmell, Byron Theron. Northern Devon District Hospital, Barnstaple, UK

\subsection{6/gutjnl-2020-bsgcampus.396}

Introduction Flexible sigmoidoscopy remains a first line test for left side colonic symptoms. However, the advantages it conferred over colonoscopy have receded. Usage in symptomatic patients has been on the wane with more sensitive stool tests, investment in colorectal services and advances in radiology. We sought to quantify post-flexible sigmoidoscopy colorectal cancer rates in symptomatic patients to determine if there is on-going utility for flexible sigmoidoscopy and provide quality assurance.

Methods We performed a retrospective review of all flexible sigmoidoscopies for symptomatic patients between March 2010 and March 2016. We used cancer registry data to identify all patients with colorectal cancer (CRC) who had had a negative flexible sigmoidoscopy in the preceding 3 years.

Patients who were diagnosed within 6 months were excluded to allow for time for linkages between databases and where there may have been additional complexity delaying the diagnosis to avoid inappropriately misclassifying patients.

Flexible sigmoidoscopies were categorised as true positive (CRC within 6 months of the index procedure) and false negative (CRC within 6-36 months). We performed a qualitative review of each false negative.

Results 7199 flexible sigmoidoscopies were done over 6 years. $232(3.2 \%)$ cases of CRC were diagnosed at index test (true positives). The false negative rate was $3.75 \%(9 / 240)$. The false negative rate was higher in women at $6.1 \%(6 / 99)$ vs $2.12 \%$ $(3 / 141)$ in men. There were no false negatives in individuals younger than 60 years. The false negative rate in those over 60 was $4.6 \%(9 / 194)$.

All but 2 of the false negative cases were proximal to the splenic flexure, 1 was beyond the extent of the flexible sigmoidoscopy, the other within a diverticular segment which had been passed. 6/9 had an indication for full colonic review. 2 had iron deficiency anaemia, 1 had a change in bowel with loss of weight and 3 had undiagnosed abdominal pain. In hindsight, it is likely that the missed cancer explained the symptoms, but it is acknowledged that the indications were of variable strength.

Conclusions Advancing age and female gender are associated with higher false negative rates. Bowel preparation did not seem to be a factor in missed CRC. Our data suggest that flexible sigmoidoscopy is a safe first line test in patients with left sided colonic symptoms as it has low false negative rate and diagnostic yield similar to other invasive investigations for alarm symptoms. However, adequate consideration of complex symptoms and safety netting should be in place to ensure there are no indications for a full colonoscopy. 